\title{
Geostatistical mapping of land prices in Taiwan Case: an empirical comparison of ordinary kriging
}

\author{
Cheng-Ya, Chang a, * \\ ${ }^{a}$ Chinese Culture University, Graduate School of Earth Science, PhD. Student, changchengya@gmail.com \\ * Corresponding author
}

Keywords: ordinary kriging, land prices, price prediction, spatial autocorrelation

\section{Abstract:}

This study discusses the use of land price data to estimate the land price using the ordinary kriging method (OK). Moreover, compares the Gaussian and exponential semivariogram model by cross-validation and which one is more suitable for the ordinary kriging model also has a good Mean absolute percentage error (MAPE). The study area is in Tainan City, Taiwan. The data sample is a land transaction case from 2012 to 2018. The study adopts the inherent hypothesis that the difference between the random variables in different spatial locations is a random variable, and the expected value and the variance are only related to the distance between the random variables and the spatial position. The ordinary kriging method was used as a tool in the study. First, the data were randomly divided into experimental group $(90 \%)$ and control group $(10 \%)$ by spatial random sampling. A total of 10 pairs were combined to ensure the stability of the verification. The space autocorrelation distance (Moran`s I) is taken as the influence range, and the semivariogram are verified by cross-validation method, and then verified by mean absolute percentage error (MAPE). The study found that under the same data sample, after cross-validation, the exponential semivariogram model has a better land price prediction effect, whether it is the verification result or the mean absolute percentage error.

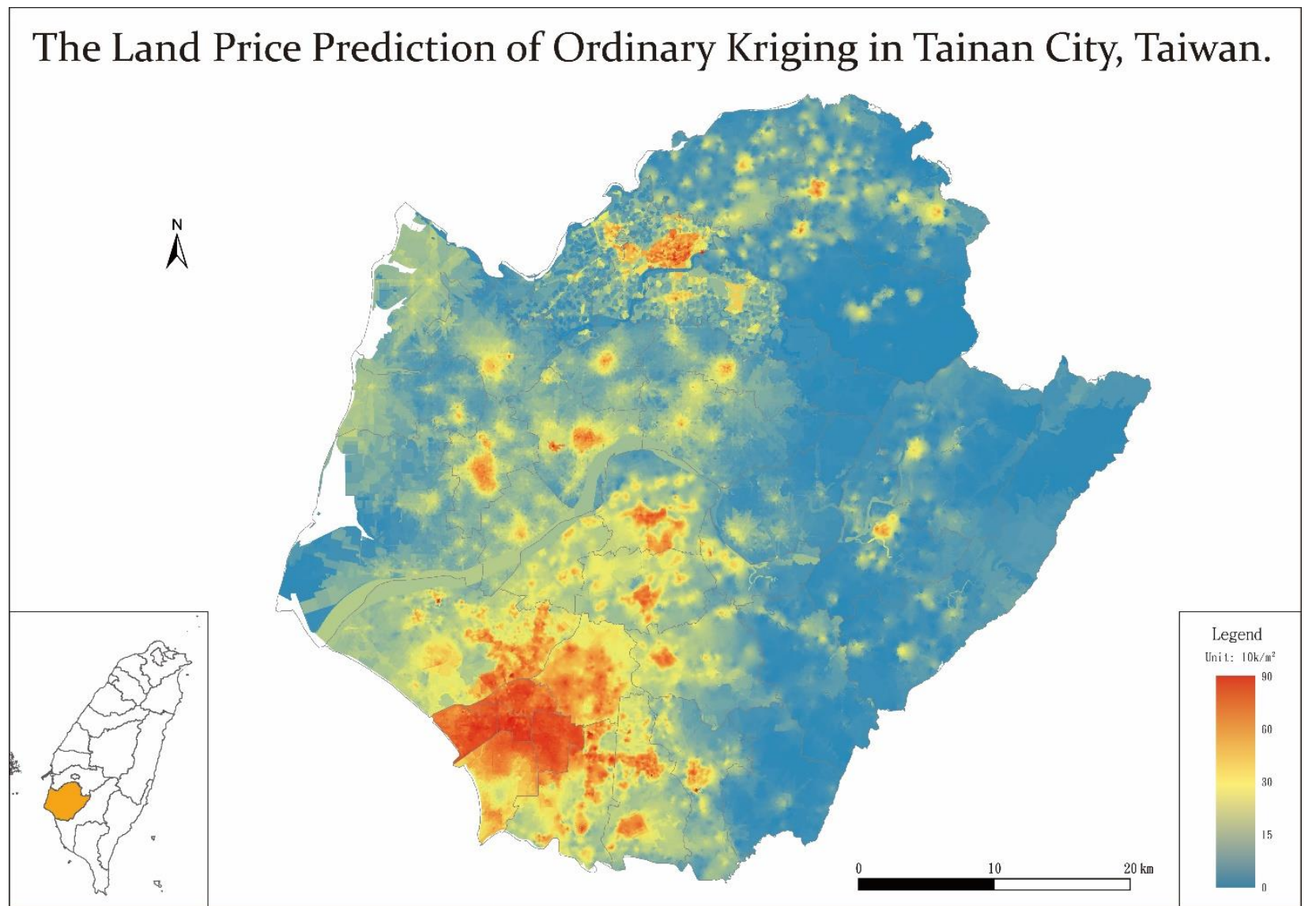

Figure 1. : The land price prediction map at Tainan City, Taiwan. 